\title{
What the Intensivists Need to Know About Critically III Myeloma Patients
}

\author{
Ranjit Nair and Krina Patel
}

\section{Contents}

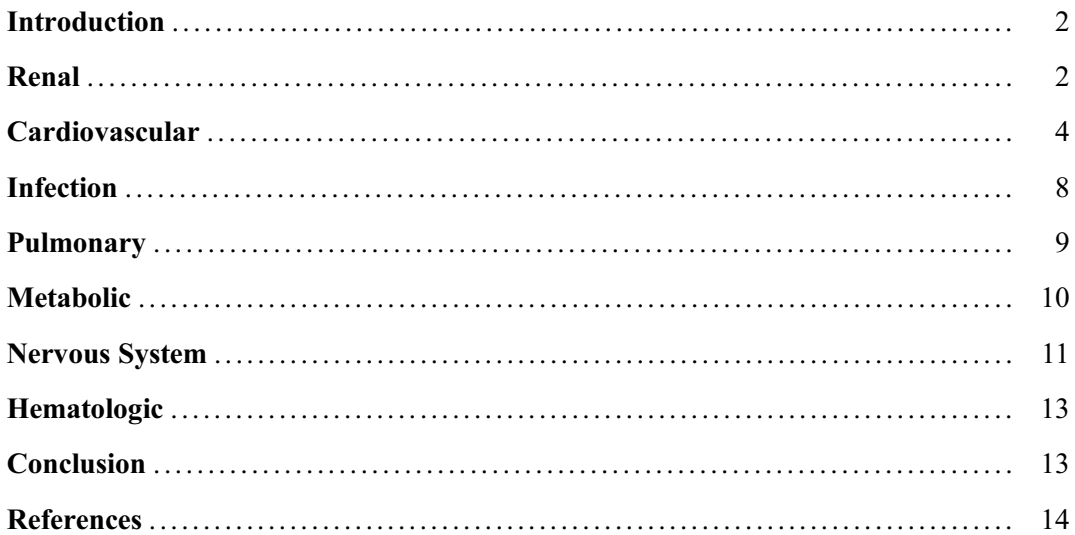

\begin{abstract}
Multiple myeloma (MM) is a hematological malignancy characterized by an increase in aberrant plasma cells in the bone marrow leading to rising monoclonal protein in serum and urine. With the introduction of novel therapies with manageable side effects, this incurable disease has evolved into a chronic disease with an acceptable quality of life for the majority of patients. Accordingly, management of acute complications is fundamental in reducing
\end{abstract}

R. Nair $\cdot$ K. Patel $(\bowtie)$

Department of Lymphoma and Myeloma, Division of Cancer Medicine, University of Texas MD Anderson Cancer Center, Houston, TX, USA

e-mail: rnair@mdanderson.org; Kpatel1@mdanderson.org the morbidity and mortality in MM. MM emergencies include symptoms and signs related directly to the disease and/or to the treatment; many organs may be involved including, but not limited to, renal, cardiovascular, neurologic, hematologic, and infectious complications. This review will focus on the numerous approaches that are aimed at managing these complications.

\section{Keywords}

Multiple myeloma $\cdot$ Oncologic emergencies 


$\begin{array}{ll}\text { Abbreviations } \\ \text { AF } & \text { Atrial fibrillation } \\ \text { ARDS } & \text { Acute respiratory distress syndrome } \\ \text { BAL } & \text { Bronchoalveolar lavage } \\ \text { DAH } & \text { Diffuse alveolar hemorrhage } \\ \text { DVT } & \text { Deep vein thrombosis } \\ \text { eGFR } & \text { Estimated glomerular filtration rate } \\ \text { FLC } & \text { Free light chain } \\ \text { IgA } & \text { Immunoglobulin A } \\ \text { IgG } & \text { Immunoglobulin G } \\ \text { IgM } & \text { Immunoglobulin M } \\ \text { LC } & \text { Light chain } \\ \text { LMM } & \text { Leptomeningeal myelomatosis } \\ \text { MM } & \text { Multiple myeloma } \\ \text { MRI } & \text { Magnetic resonance imaging } \\ \text { PCR } & \text { Polymerase chain reaction } \\ \text { PE } & \text { Pulmonary embolism } \\ \text { RIFLE } & \text { Risk of renal injury/injury to the kid- } \\ & \text { ney/failure of kidney function/loss of } \\ & \text { kidney function/end-stage disease } \\ \text { RT } & \text { Radiation therapy } \\ \text { RV } & \text { Right ventricle } \\ \text { sCr } & \text { Serum creatinine } \\ \text { TLS } & \text { Tumor lysis syndrome } \\ \text { TPE } & \text { Therapeutic plasma exchange } \\ & \end{array}$

\section{Introduction}

MM is a hematological malignancy characterized by an increase in aberrant plasma cells in the bone marrow leading to rising monoclonal proteins in the serum and urine. In general, it is a disease of older adults; however, MM may effect younger patients as well: $15 \%$ are $<50$ years, $3 \%$ are $<40$ years, and $0.2 \%$ are $<30$ years in age [74]. It accounts for about $10 \%$ of hematological malignancies in the United States, with an estimated 30,770 new cases in 2018. Patients often present with osteolytic bone lesions, fractures, bone pain, progressive anemia, hypercalcemia, renal insufficiency, recurrent infections, and/or bleeding. If not treated appropriately and urgently, any of these may lead to death. The introduction of novel agents to the traditional arsenal of drugs comprising of steroids, chemotherapy, and autologous stem cell transplantation has shifted the treatment platform from one which often required repeated acute interventions to that of a more chronic manageable disease. As the acute complications can be potentially fatal, early recognition and intensive care management is the key to successful outcomes. These patients who present with complications are often excluded from MM trials, and their prognosis in the modern era is uncertain.

Reports suggest that in-hospital mortality from acute complications of MM has decreased significantly over the past decade. Continued risk factors for death in this group of patients are organ failure, poor chronic health status, and delayed intensive care management [98]. Despite having increased life expectancy now, the majority of MM patients ultimately develop resistant subclones, leading to disease progression and disease-related complications. With increasing complexity of MM treatments, patients are at risk for more challenging drug toxicities. Recognizing and managing the potential side effects of present-day novel regimens is therefore a cornerstone in MM care. In this chapter, we will provide a systematic review of the key critical clinical events which cause medical emergencies in MM. This crucial, fundamental knowledge for all clinicians who routinely care for MM including intensivists will allow for optimal treatment outcomes and survival in MM. Complications resulting from stem cell transplant or newer therapies including chimeric antigen receptor $\mathrm{T}$ cell therapy are beyond the scope of this review and will not be discussed here.

\section{Renal}

Renal insufficiency in $\mathrm{MM}$ is a medical emergency that needs immediate work-up and management. There is a wide variation in data reporting on the incidence and the degree of renal failure due to differences in the definitions of renal dysfunction, sample sizes of studies, and populations assessed. Renal dysfunction occurs in more than a third to half of newly diagnosed patients (serum creatinine $(\mathrm{sCr})$ above the upper normal limit or $2 \mathrm{mg} / \mathrm{dL}$ or eGFR $<60 \mathrm{~mL} / \mathrm{min} / 1.73 \mathrm{~m}^{2}$ ). Table 1 details the list of common causes of 
Table 1 Pathophysiology of renal disease in multiple myeloma

\begin{tabular}{|c|c|}
\hline $\begin{array}{l}\text { Causes of renal disease in } \mathrm{MM} \text { - monoclonal protein } \\
\text { dependent }\end{array}$ & $\begin{array}{l}\text { Causes of renal disease in } \mathrm{MM} \text { - monoclonal protein } \\
\text { independent }\end{array}$ \\
\hline Cast nephropathy (MM kidney) & Hypercalcemia \\
\hline Monoclonal immunoglobulin deposition disease & Nephrotoxic drugs \\
\hline Light chain amyloidosis & Infections \\
\hline $\begin{array}{l}\text { Glomerulonephritis } \\
\text { Cryoglobulinemic glomerulonephritis } \\
\text { C3 glomerulonephritis } \\
\text { Mesangial proliferative glomerulonephritis } \\
\text { Immunotactoid glomerulonephritis }\end{array}$ & Anti-MM drug induced \\
\hline Fanconi syndrome & Iodinated contrast \\
\hline $\begin{array}{l}\text { Glomerulopathy } \\
\text { Minimal change disease } \\
\text { Membraneous }\end{array}$ & \\
\hline
\end{tabular}

renal dysfunction encountered in clinical practice. As per the RIFLE risk category, approximately $10-15 \%$ of patients develop renal failure, and half become dialysis dependent [9, 64]. Even after an initial renal recovery with appropriate myeloma-directed therapy, more than half of patients can still develop renal failure during the disease course. In the absence of renal recovery, mortality risk is reported to be higher in this population with shortened overall survival. With novel therapies in myeloma, reversibility of renal insufficiency (RI) is associated with improved survival with some studies demonstrating similar survival in patients who recover from acute kidney injury (AKI) and those without renal impairment. Therefore, achieving renal recovery is of priority and the main goal in these patients [1, 11, 29, 43, 125].

MM kidney: In many cases (approximately $40-50 \%$ of cases), renal damage is caused by the disproportionate free light chain (FLC) excretion through kidney glomeruli, buildup of light chains in distal tubules, and cast formation which further leads to renal tubular obstruction, an entity referred to as MM kidney. The tubules eventually rupture and FLC extravasates, causing interstitial inflammation and damage. This is usually compounded by renal injury from hypercalcemia, dehydration, poor intake, use of nephrotoxic analgesics, IV contrast, use of ACE/ARB blockade, underlying chronic kidney disorder, and/or underlying infection.
Early diagnosis and timely therapeutic intervention at the time of presentation to the emergency center are critical. Therapy should include aggressive resuscitation with IV fluids in efforts to maintain normal urine output, treating concomitant hypercalcemia and other electrolyte imbalances, initiating treatment of underlying infection, and avoiding nephrotoxins. A multidisciplinary approach with hematology and nephrology consults is paramount to optimize care in these patients. Suspicion of cast nephropathy in newly diagnosed myeloma patients with serum FLC above $1000 \mathrm{mg} / \mathrm{L}$ should be high, especially if associated selective proteinuria composed of FLC/BJP exists, as the degree of renal injury is usually related to tumor load [55]. A percutaneous kidney biopsy with histology is not required to initiate treatment if myeloma has been confirmed but can be helpful to confirm the diagnosis, assess concomitant light chain or amyloid deposition disease, and predict prognosis. The need for a renal biopsy should be discussed for patients with lower levels of toxic FLC, as these patients are more likely to have alternate etiologies for their RI. In patients with significant light chain burden, it is critical to begin treatment immediately with renal recovery as the major immediate goal.

The only proven modality with the best chance of renal recovery is rapid cytoreduction to decrease light chain production. Newer chemotherapy agents lower FLCs quickly, especially with a combinatorial approach. Many clinical 
trials have shown that a bortezomibdexamethasone combination regimen achieves this goal [30]. Attaining a pre-dialysis serumfree light chain level below $500 \mathrm{mg} / \mathrm{L}$ and a $60 \%$ reduction after the first cycle of chemotherapy are independent predictive factors of renal recovery and hemodialysis independence at 1 year $[56,57]$.

Hemodialysis or TPE for the sole purpose of offloading tumor burden is currently investigational, as none of the randomized trials have shown a significant benefit. Though it takes a few days to weeks to observe the impact of chemotherapy on light chain production, current evidence suggests using hemodialysis only for clinical indications of renal replacement therapy which may exist and not solely for reduction of tumor load. Based on two randomized clinical trials, the use of high cutoff hemodialysis to rapidly reduce the load of nephrotoxic light chains may be an option in patients requiring dialysis when treated with bortezomib-based therapies, but the results may be stochastic at best $[14,22$, 108]. TPE can rapidly reduce FLC loads; however its role in cast nephropathy comes from small retrospective trials which provide no data on the renal histology or SFLC levels. In studies that demonstrated benefit, obtaining a biopsy and using a proteasome inhibitor (PI)-based treatment were not uniformly applied, making interpretation of the data difficult. Especially in the era of novel drugs, the evidence in favor of TPE is limited $[21,29,63]$ unless there is evidence of $\operatorname{IgM}$ or IgA paraproteinemia-associated hyperviscosity syndrome. The utility of urinary alkalization is debated as this theoretically decreases tubular cast formation and, however, can cause risk of renal calcium precipitation, especially if the patient presents with concurrent hypercalcemia.

Acute tubular necrosis: ATN is a consequence of an already compromised renal function in patients with cast nephropathy. Hypercalcemiarelated vasoconstriction with subsequent reduced renovascular flow, dehydration, and use of loop diuretics can speed up cast formation.

There are some reports of carfilzomib causing direct proximal tubular injury; however, the risk appears to be very small [70].
Monoclonal deposition disease: In contrast to myeloma kidney, MIDD (heavy chain, light chain, or heavy chain-light chain) and AL amyloidosis result from gradual non-fibrillar (Congo red negative) and fibrillar (Congo red positive) protein deposition, respectively, in the glomeruli. MIDD is most commonly kappa subtype, while AL amyloidosis tends to be lambda; both tend to be associated with predominant albuminuria unlike cast nephropathy. Though the most characteristic presentation is nephrotic syndrome due to glomerular involvement, patients with MIDD can have rapid deterioration of renal function which could be fatal if diagnosis is not reached in a patient presenting with new-onset renal failure. Life-threatening complications which need to be closely monitored include arrhythmias, congestive heart failure, and bleeding diathesis. To confirm the diagnosis, subcutaneous fat pad biopsy can be pursued initially, as it is less invasive and can potentially diagnose an AL amyloid deposition disease. If negative, proceed with kidney biopsy to look for AL amyloid, MIDD, or other potential renal glomerulopathy.

Fanconi syndrome: The presence of hypophosphatemia, hypokalemia, hypouricemia, renal tubular acidosis, and glycosuria should raise the suspicion of Fanconi syndrome in MM. It is a rare wasting syndrome due to proximal renal tubule dysfunction more commonly seen in patients with MGUS leading to loss of glucose, phosphate, amino acids, and uric acid. Though it runs a benign course, it can complicate the concomitant electrolyte imbalance and renal failure. Ironically, despite the glycosuria, the serum glucose is usually normal, which goes along with the defect in the proximal tubular transport defect [15].

\section{Cardiovascular}

MM patients are at high risk for cardiac complications secondary multiple factors including an older age group of patients with underlying comorbidities, concurrent kidney involvement, MM-associated deposition disease, and/or antiMM drug-related side effects. 
Cardiac amyloid: Approximately 10\% of patients with $\mathrm{MM}$ can have concomitant light chain (AL) amyloidosis, the most common type of systemic amyloidosis associated with plasma cell dyscrasias; often this association is missed at the time of MM diagnosis. In amyloidosis, heart involvement is seen in approximately half of the cases [32]. Cardiac amyloidosis can be clinically silent initially, and a MM patient presenting with progressive dyspnea, worsening edema with evidence of heart failure, or dysrhythmia presenting as syncope or hypotensive event requires a thorough work-up to rule out coexisting amyloid disease. Detection of amyloid in any organ requires ruling out cardiac involvement. Other symptoms which warrant amyloid work-up include easy bruising, periorbital purpura, macroglossia (which is better appreciated when associated with tooth indentation), and carpal tunnel syndrome. Once significant symptoms of cardiac amyloid appear, most commonly related to heart failure, median survival shortens, especially in the event of a late diagnosis. Amyloid deposition takes place extracellularly throughout the ventricles, atria, valves, conduction system, and in the perivascular regions of small vessels. This eventually leads to thickened, non-dilated ventricles as well as atrial thickening that eventually progresses to thinned dilated atria.

The serum concentration of free light chains and cardiac enzymes ( $\mathrm{N}$-terminal pro-brain natriuretic peptide and cardiac troponins) are sensitive markers for systemic and cardiac involvement, respectively, in AL amyloidosis [116]. N-terminal pro-B-type natriuretic peptide levels are elevated in cardiac amyloidosis, even in the absence of heart failure, due to regional myocardial stress due to fibrillar extracellular deposition [90]. Cardiac troponins can be elevated, even with an unremarkable coronary angiography, due to predominant deposition in the small intramural vessels sparing the epicardial arteries, but associated with nonischemic destruction of cardiomyocytes. ECG findings suggestive of cardiac involvement include a pattern of low voltage (cardiac changes from amyloid infiltration rather than myocyte hypertrophy) defined as a QRS voltage amplitude of $\leq 0.5 \mathrm{mV}$ in all limb leads or $\leq 1 \mathrm{mV}$ in all precordial leads (sensitivity 45-70\%). This may be described as a pseudo-infarct pattern (50-75\%). Atrioventricular right- or left-bundle branch blocks may also suggest cardiac amyloid. Echocardiography in advanced stages usually demonstrates a restrictive pattern due to the structural changes and increased echogenicity of the myocardium with a "granular speckling" (low sensitivity of $26-36 \%$ but with a high specificity (71-81\%)). Cardiac MRI in advanced cardiac amyloidosis shows diffuse subendocardial heterogeneous enhancement in a nonvascular distribution on delayed contrast-enhanced MR images using inversion recovery technique. This, along with diffuse multichambered wall thickening, posterior right atrial wall thickening, or interatrial septal thickening $>6 \mathrm{~mm}$, is specific to amyloidosis $[10,112,126]$. Cardiac catheterization is not always required but used to obtain endomyocardial biopsy. It may help to provide important information for cardiac hemodynamics, which generally shows impaired ventricular filling with an elevated left ventricular end-diastolic pressure. Demonstration of left ventricular diastolic pressure greater than right remains to distinguish restrictive cardiomyopathy from its differential diagnosis of constrictive pericarditis. Fine-needle aspiration of abdominal fat is relatively sensitive for amyloid deposits $(>70 \%$ of patients with AL amyloidosis); other sites of amyloid deposition include minor salivary glands, gingiva, and rectum [3, 69]. In a patient with clinical suspicion of cardiac involvement, the presence of physical exam findings, blood markers, imaging, and a cardiac biopsy consistent with amyloid are adequate for diagnosis.

Patients presenting with acute heart failure in AL amyloidosis require intensive care monitoring and aggressive supportive care. Diuresis is the key and is probably the only treatment modality that can improve hemodynamics. Optimal therapy usually requires using higher doses of diuretics or in combinations (furosemide, torsemide, spironolactone, and metolazone) to maintain adequate diuresis, especially in the setting of nephrotic syndrome. An intravenous route is likely preferred in the acute stage, due to the often-present gut edema and better bioavailability. 
Aggressive supportive care includes maintaining strict intake output measurements, dietary salt restriction, measuring daily weights, and monitoring for arrhythmias. The uses of cardiac protective drugs such as beta-blockers should be reserved for cases of atrial fibrillation to maintain adequate filling pressure. In patients without $\mathrm{AF}$, its use should be discussed on a case-by-case basis, considering the significant risks including labile blood pressure due to autonomic neuropathy and risk of bradycardia due to coexistent conduction defects. Caution should be maintained with the use of angiotensin-converting enzyme inhibitors and angiotensin II inhibitors, due to similar risk of exacerbating hypotension. Both digoxin and calcium blockers, especially nifedipine, have the tendency to bind to amyloid fibrils. This can precipitate congestive heart failure due to increased susceptibility to digoxin toxicity and enhance the negative ionotropic effect with calcium channel blockers [41, 84, 107].

Syncope can result from AF with rapid heart rate, embolic phenomenon, conduction defects, bradycardia, and ventricular arrhythmias. The most common atrial arrhythmia (10-15\% of patients) is $\mathrm{AF}$ and is associated with a very high incidence of thromboembolism. In a Mayo Clinic study, cardiac amyloidosis patients undergoing transesophageal echocardiography had a higher frequency of intracardiac thrombus (35\%) irrespective of the presence of atrial fibrillation. Factors associated with increased risk of intracardiac thrombosis include atrial fibrillation, poor left ventricular diastolic function (grade 3 or 4), lower left atrial appendage emptying velocity $(\leq 15 \mathrm{~cm} / \mathrm{s})$, increased RV wall thickness, low systolic blood pressure, and increased heart rate. This group of patients should be considered high risk for thromboembolic events and merits screening with TEE. Anticoagulation should be strongly considered in the presence of any of these risk factors due to the heightened risk for mural thrombus development and embolic events. This should be weighed against the risk of bleeding in these patients due to the potential for concomitant coagulopathy and fragile vasculature [36].

Pacemakers are a consideration in the presence of heart block or symptomatic bradycardia. The role of intracardiac defibrillators is controversial due to limited evidence showing a survival benefit both for primary and secondary prevention. Several studies report ventricular arrhythmias in about a third of patients; however, this does not appear to be the leading cause of mortality in this patient group. Once congestive heart failure occurs, the clinical course for patients rapidly deteriorates, with a median survival of 6-12 months from diagnosis. Death in severe cardiac amyloid is often heralded by agonal bradycardia, followed by electromechanical dissociation or PEA. Nonetheless consensus guidelines do not support ICD placement for primary prevention of SCD in patients if life expectancy is $<1$ year. Hence these devices have rarely been used in patients with cardiac AL amyloidosis. With improvement in the therapeutic platform of cardiac amyloidosis and improved survival, it becomes a priority to be able to select the patients that benefit from antiarrhythmic prophylaxis and primary prevention ICDs. With the available data, ICDs should be considered in cardiac amyloid patients with recurrent episodes of non-sustained VT, unexplained syncope, young age at diagnosis, early stages of disease at diagnosis with low levels of cardiac biomarkers, and prognosis $>1$ year $[47,48,71,127]$.

Anti-myeloma drug-related cardiotoxicity: Proteasome inhibitors are currently the main class of drugs used in front line and in the relapsed setting of MM. FDA-approved therapies in this class include bortezomib, carfilzomib, and ixazomib [93]. Among them, carfilzomib has shown the highest rates of cardiotoxicity. In a meta-analysis looking at the cardiotoxicity of carfilzomib, all-grade and high-grade cardiotoxicity were seen in $18 \%$ and $8 \%$ of patients, respectively, higher than the control arm. The most significant events included heart failure ( $\sim 5 \%)$, arrhythmias $(\sim 2.5 \%)$, and ischemic events $(\sim 2 \%)$. Rare reports of carfilzomibassociated cardiac arrests also exist. Risk can be higher in patients with comorbidities such as older age, obesity, anemia, kidney dysfunction, previous mediastinal radiation, high-dose corticosteroids, prior or concurrent anthracycline exposure, use of higher carfilzomib doses, short 
infusion times, and undiagnosed amyloidosis $[5,52,92,133,135]$. Anecdotal reports suggest bortezomib may lead to cardiotoxicity as well, although a recent meta-analysis did not find a significantly increased risk compared to the control arm [128, 136]. The first intervention in suspected cardiotoxicity is to hold further therapy and follow expectant management. In cases of congestive heart failure, once the heart failure symptoms improve, it is reasonable to restart the chemotherapy at a lower dose, with a longer infusion time and limiting peri-treatment IV fluids. Arrhythmic events would require careful consideration regarding rechallenging patients after considering the risk-benefit ratio.

Hyperviscosity syndrome: In a patient undergoing work-up for $\mathrm{MM}$ or with an established diagnosis, symptoms of headache, nose bleeds, mental status or visual changes, auditory symptoms, focal weakness, excessive lethargy, seizures, or coma should raise suspicion for hyperviscosity syndrome. Though extremely uncommon in $\mathrm{MM}(\sim 5 \%)$ in comparison to Waldenström's macroglobulinemia (10-30\%), a low threshold should be kept to work up a patient for hyperviscosity as this can be fatal if not recognized early. The viscosity tends to increase with increase in molecular size, shape, and weight of the involved paraprotein and abnormal polymerization. Patients tend to develop symptoms when the serum viscosity reaches 4 centi-Poise (cp) (normal serum viscosity, $1.4-1.8 \mathrm{cp}$ ). This usually corresponds to an $\mathrm{M}$ spike of $3 \mathrm{~g} / \mathrm{dL}$ if $\mathrm{IgM}, 4 \mathrm{~g} / \mathrm{dL}$ for $\mathrm{IgG}$, and 6-7 g/dL for IgA paraproteinemia. However, these levels are arbitrary, and the relationship is nonlinear as patients can have symptoms even with a level lower or could be asymptomatic with a higher level $[49,94]$. In MM, IgG (mostly IgG3) or IgA paraprotein tends to be reported more commonly with hyperviscosity than $\operatorname{IgM}$ most likely due to the inherent rarity of $\operatorname{IgM}$ MM [28, 81].

Due to increased serum viscosity, there is impaired blood flow and sludging throughout the microvasculature, leading to tissue ischemia, placing the patient at risk for thrombosis to major organs leading to myocardial, cerebrovascular, and retinal ischemia. With passive venous congestion, patients can often present with bleeding symptoms. Thorough physical exam is paramount including a fundoscopic exam, which may reveal dilated, tortuous, engorged, "sausage" veins with associated hemorrhages. Bleeding at sites involving mucosa is common which can include oral and nasal cavity, the uterus, and the gastrointestinal tract. Purpura and skin bruising are also common.

Consensus MM guidelines do not recommend plasmapheresis in the absence of signs or symptoms for hyperviscosity. However, a low threshold should be maintained to initiate treatment at the earliest suspicion of symptoms or signs; it follows a two-prong strategy consisting of immediate plasmapheresis to lower the excess paraproteins and chemotherapy directed at the primary process of MM. Unlike the response of IgM-related paraproteinemia ( $>80 \%$ intravascular), where a $50-60 \%$ reduction of viscosity is expected with one treatment, $\operatorname{IgA}$ or $\operatorname{IgG}(<50 \%$ intravascular) hyperviscosity can take several sessions to achieve the same result [83]. If plasmapheresis is not readily available and the patient is having lifethreatening symptoms, phlebotomy may reduce hyperviscosity-related acute symptoms. In MM patients, who are often anemic at presentation, blood transfusions should be done only in emergent situations and with caution until after the initiation of plasmapheresis to prevent rise in serum viscosity $[110,119]$.

Venous thromboembolism: Patients with $\mathrm{MM}$ are at an increased risk of venous thrombosis due to patient-related, disease-related, and treatment-related factors. Patient-related factors include older age, recent surgery or immobilization, and decreased functional status; the major disease-related factor is increased pro-thrombotic status from heightened inflammatory markers, especially at diagnosis. There is a high level of factor VIII and von Willebrand factor as well as acquired resistance to activated protein $\mathrm{C}$ [33]. In addition, MM patients treated with immunomodulators especially thalidomide or lenalidomide used in combination with steroids or other chemotherapeutic agents increase the risk further [89, 132]. Management of acute DVT or PE involves withholding the offending drug 
if any and starting immediate systemic anticoagulation with low-molecular-weight heparin or unfractionated heparin. LMWH is the preferred anticoagulation at the time of transition to outpatient therapy as evidence suggests better safety and a superior efficacy in stabilizing the clot in comparison to other anticoagulants [67]. Factors that make oral anticoagulation less ideal is drugdrug interaction and unreliable therapeutic levels resulting from patient-related malnutrition, infection, and drug holds from cytopenias. Once full anticoagulation is attained, the immunomodulator can be reintroduced.

In the presence of thrombocytopenia, which is commonly encountered in MM patients, the dose of LMWH needs adjustment and in general a recommendation of $50 \%$ dose reduction if platelet counts below $50,000 / \mu \mathrm{L}$, and discontinuation if platelet count $<20,000 / \mu \mathrm{L}$ is followed. Duration of anticoagulation should be a minimum of 6 months; however in the presence of the offending drug, systemic anticoagulation should be continued for the total duration of therapy [13]. Arterial thromboembolic events, though rare, have also been reported with thalidomide, lenalidomide, and pomalidomide; rarely, this may present as myocardial infarction or stroke. The role of newer oral anticoagulants including factor $\mathrm{Xa}$ inhibitors and direct thrombin inhibitors is currently investigational in the treatment of malignancy-associated thromboembolic events; however, they are a good alternative for patients with renal failure [26].

\section{Infection}

Sepsis/invasive infections: $10-15 \%$ of newly diagnosed MM patients succumb to the disease in the first few months with infection contributing to more than half the cases $[6,53,88]$. High incidence of early infection in MM is due to the suppressed humoral and T cell-mediated immunity by the disease itself. Immunosuppression is mediated by disease- and treatment-related factors including decreased ratio of functional to dysfunctional immunoglobulins, defects in antibody opsonization, steroid-related $\mathrm{T}$ cell defects, secondary immunodeficiency related to chemotherapy, restricted pulmonary reserve from thoracic rib fractures and opiate use, mucosal damage, indwelling catheters, and presence of renal failure [53, 88, 100]. Bacterial infections, especially Streptococcus pneumoniae, Haemophilus influenzae, and Escherichia coli, tend to be the predominant organisms causing infection at diagnosis, while Staphylococcus aureus and other gram-negative infections are more common in the relapsed refractory setting $[109,114]$. The use of novel drugs combined with steroids has increased the rate of viral and fungal infections which can occur any time during the disease course [12, 58, 91]. Viral infections, especially herpes simplex virus (HSV) and varicellazoster virus (VZV), have increased in incidence with the introduction of proteasome inhibitors such as bortezomib. Viral prophylaxis with acyclovir or valacyclovir is recommended in all patients undergoing therapy with proteasome inhibitors or monoclonal antibodies. Although invasive fungal infection (approximately 2-3\%) incidence is low, the mortality rate is high with approximately $60 \%$ of patients requiring ICU management. The introduction of novel agents tends to be associated with later-onset invasive fungal infections compared to studies from the conventional chemotherapy era where an earlier onset was noted [122]. Furthermore the use of long-term and high-dose dexamethasone can cause persistent defect in T cell-mediated immunity which predisposes patients to mucosal candidiasis, $P$. jirovecii, HSV, and VZV infections.

Following sepsis guidelines with aggressive fluid resuscitation and broad-spectrum antibiotic coverage as well as growth factor support if neutropenic is warranted in a patient presenting with suspected sepsis/infection. In case of clinical deterioration with unclear source of infection, persistent febrile neutropenia, or hemodynamic compromise, consider computer tomography (CT) scan including sinus imaging. Other investigative modalities for diagnosis should include bronchoscopy/BAL with microscopy and bacteria, viral, and fungal culture. In suspected cases of fungal infection, Aspergillus PCR and galactomannan testing on serum and BAL should 
be performed. Due to concomitant immunodeficiency, treatment with intravenous immunoglobulins is considered in patients with recurrent bacterial or viral infections; however its role in treating an acute infection is limited.

\section{Pulmonary}

Direct involvement of pulmonary parenchyma in MM is extremely rare. Pulmonary complications can arise from a broad range of different mechanisms both infectious and noninfectious. In majority of the cases presenting with respiratory failure, the etiology is infectious. Patients with myeloma can present with lung infiltrates, ground glass opacities, nodules, masses, effusion, and lymphadenopathy. Noninfectious causes that need to be ruled out include congestive heart failure, pulmonary embolism, pulmonary hemorrhage, and drug-induced pneumonitis.

Myeloma lung: Case reports and case series describe the various ways MM can involve the lung. Presentation of lung disease is variable and includes consolidative or interstitial pattern infiltrates on imaging, intraparenchymal plasmacytomas, intrapulmonary calcifications, or pleural effusions [60, 73, 139]. Presentations similar to ARDS have also been reported $[40,80,99,106,134]$. A high index of suspicion is required as there is a broad differential of diagnoses with similar radiographic findings. Patients with extramedullary MM tend to have more aggressive disease and can deteriorate rapidly due to disease progression if not recognized and treated early. If there is evidence of MM progression on blood tests or bone marrow, a biopsy (surgical, transbronchial) or bronchoscopy with aspiration and/or bronchoalveolar lavage (BAL) should be strongly considered with microbiologic and flow cytometric analysis. Once infection is excluded and evidence of progression is confirmed, prompt initiation of MM directed therapy is warranted [62].

Diffuse alveolar hemorrhage: In patients presenting with acute onset hypoxia, pulmonary opacities, and fevers, especially in the setting of thrombocytopenia and anemia, pulmonary hemorrhage is a possibility. Diffuse alveolar hemorrhage (DAH) has been rarely reported in MM patients, but there have been cases associated with bortezomib and in the posttransplant setting $[2,7$, $8,120]$. Though rare, this is potentially a fatal event. A thorough microbiology screen, echocardiogram, CT imaging, autoimmune work-up, and coagulopathy work-up are warranted [115]. BAL showing presence of gross blood and the percentage of hemosiderin-laden macrophages (siderophages) $>20 \%$ at BAL are suggestive of a diagnosis of DAH [25, 42, 118]. Aggressive supportive care and empiric broad-spectrum antimicrobial therapy should be considered. The role of early initiation of steroids is currently based on retrospective series and case reports. An MD Anderson prospective study examined 119 critically ill adult hematopoietic transplant patients treated for $\mathrm{DAH}$, and on multivariate analysis, an initial medium (250-1000 mg/day) and highdose $(1000 \mathrm{mg} /$ day $)$ steroids were associated with a higher ICU mortality $(P=0.01)$ as compared with the low dose $(<250 \mathrm{mg} /$ day $)$. Adjunctive treatment with aminocaproic acid in this study, which is commonly used in DAH, did not produce differences in outcomes [85, 105].

Drug-induced interstitial pneumonitis (DIP): DIP manifesting as interstitial lung disease has been reported with lenalidomide, thalidomide, and bortezomib [16, 20, 113, 123]. Though mechanisms are not fully elucidated, oxidative stress, prostaglandin E2 (PGE2) inhibition, fibroblast proliferation, and impairment of cell repair by epidermal growth factor receptor blocking may play a role. BAL with culture and microscopy should be able to help rule out infectious causes. The lymphocytic preponderance typically seen in bronchoalveolar lavage (BAL) is supportive of a hypersensitivity mechanism; however, this finding is nondiagnostic and requires meticulous exclusion of other causes. Invasive procedures like biopsy usually do not provide any specific findings diagnostic for DIP and, therefore, should be obtained only on a case-by-case basis. Once DIP is suspected, stopping the offending agent is the main therapeutic approach; steroids may be used in severe cases [4, 61]. Although no guidelines exist on the duration of steroids, in general, a 
slow taper is recommended [61]. Caution should be maintained on rechallenging the patients with the offending medication as the risk of recurrent reaction is extremely high.

\section{Metabolic}

Hypercalcemia: Being one of the four diagnostic criteria for MM (CRAB criteria - calcium, renal insufficiency, anemia, and bone disease), hypercalcemia is defined as calcium level corrected for albumin $\geq 11 \mathrm{mg} / \mathrm{dL}$. Almost a third of MM patients are diagnosed with hypercalcemia at diagnosis or during the disease course $[64,141]$. The mechanism is due to local osteolysis; at the cellular level, it is mediated by interleukin 1 , interleukin 6 , and tumor necrosis factor $\alpha$ with RANKL being the final common mediator. Most patients presenting with hypercalcemia have associated anemia, thrombocytopenia, kidney dysfunction, lytic lesions, and advancedstage disease. Hypercalcemia is associated with aggressive disease biology and heavy disease burden and remains a poor prognostic feature even in the era of novel agents [141].

Clinically, hypercalcemia can be asymptomatic; however, patients often have symptoms which range from subtle signs of malaise or fatigue to severe consequences including renal failure, altered mentation, seizures, shortened QT, and risk of arrhythmias. Gastrointestinal manifestations such as nausea, vomiting, ileus, anorexia, and episodes of pancreatitis have been reported [82]. If not treated promptly, hypercalcemia is lethal. Increased calcium promotes natriuresis which can further worsen an already compromised kidney function. This dehydration then leads to worsening hypercalcemia and renal function.

Though the threshold levels are arbitrary, patients with serum calcium level $<12 \mathrm{mg} / \mathrm{dL}$ or minimal symptoms can be managed with fluid resuscitation which reverses the hypovolemia, repletes the intravascular volume deficit, and improves urinary calcium excretion. Though the definitive approach is prompt MM treatment initiation, often due to patient frailty and concomitant organ damage, chemotherapy cannot be initiated until the patient is more stable. If the patient is on calcium, vitamin D supplements, thiazide diuretics, or any other drugs which promote hypercalcemia, they should be stopped immediately. In patients with levels $>14 \mathrm{mg} / \mathrm{dL}$ or with significant symptoms, other adjunct therapies should be used as a temporizing measure. Loop diuretics, which were previously commonly used for hypercalcemia, should not be routinely used. The utility of forced saline diuresis is not consistent; with lower doses, calciuresis is an indirect effect of the accompanying natriuresis. To cause a direct calciuretic effect, doses as high as $100 \mathrm{mg} / \mathrm{h}$ is required, which can cause unwanted metabolic derangements and worsening renal cast formation with risk of worsening renal dysfunction [68]. The use of calcitonin to treat patients with hypercalcemia is limited owing to its inability to dramatically lower serum calcium levels. However, it can work synergistically with other therapies without significant adverse side effects until chemotherapy can be initiated. Calcitonin, by inhibition of bone resorption and increased renal calcium excretion, has rapid onset of action and can lower calcium levels by $1-2 \mathrm{mg} / \mathrm{dL}(0.5 \mathrm{mmol} / \mathrm{L})$. The dose is $4 \mathrm{IU} / \mathrm{kg}$ given subcutaneously or intramuscularly every 6-12 h. The efficacy in general wears off after 2-3 days due to the escape phenomenon (tachyphylaxis) of the calcitonin sensing receptors [65]. Preclinical studies suggest tachyphylaxis can be prevented with the addition of corticosteroids [27, 65, 129]. With its anti-MM effect, adding corticosteroids can add to the synergy to this combination in the treatment of hypercalcemia.

The role of steroids in hypercalcemia of malignancy comes from case reports and case series. Corticosteroids decrease calcium absorption from the gut, decrease renal tubular reabsorption, and promote excretion [24, 39]. Prednisone (dose of $20-40 \mathrm{mg} /$ day) or IV hydrocortisone (dose of $200-300 \mathrm{mg} /$ day) for 3-5 days is usually used in this setting. This dose, which is effective in patients with chronic granulomatous diseases (e.g., sarcoidosis) and malignancies such as lymphoma, may be suboptimal for patients with MM 
due to the direct osteolytic action of MM cells. If clinical status permits, dexamethasone should be considered at MM treatment doses ranging from 20 to $40 \mathrm{mg}$ weekly or 20 to $40 \mathrm{mg}$ daily on a 4 days on, 4 days off schedule.

Intravenous bisphosphonates (pamidronate, zoledronic acid, ibandronate) work by blocking osteoclast-mediated bone resorption. Intravenous zoledronic acid is more routinely used than other drugs in this category due to its higher potency, efficacy, and shorter infusion time (15 min), when compared to ibandronate and pamidronate [76, 96, 97, 111]. Bisphosphonates have an established role in severe or symptomatic hypercalcemia, but often it is challenging to administer them upfront for MM patients due to the renal function-based dose limitation [19]. It also has slow onset of action and reaches peak in about 2-5 days; therefore it needs to be used in a combinatorial approach. Denosumab, on the other hand, has a renally independent clearance mechanism and hence does not require kidney functionbased dosing adjustments. It is currently FDA approved to treat hypercalcemia of malignancy refractory to bisphosphonate therapy and is dosed at $120 \mathrm{mg}$ subcutaneous injection every 4 weeks with additional doses of $120 \mathrm{mg}$ on days 8 and 15 of the first month of therapy $[37,50]$. Mithramycin and gallium nitrate are drugs of historical interest and are not currently used in the standard setting. In refractory cases of hypercalcemia seen in advanced MM and kidney involvement, with impending cardiovascular or cerebrovascular deterioration, hemodialysis $\mathrm{Ca}^{2+}$-free dialysate should be strongly considered.

Tumor lysis syndrome: Due to the low proliferative activity of plasma cells which is $<10 \%$ in majority of patients, TLS is extremely uncommon in MM $[18,79]$. MM patients who are likely to be at risk are patients with high tumor burden (as noted by an increased serum lactate dehydrogenase and beta-2 microglobulin), diffuse bone marrow disease (plasma cells $>70 \%$ of all nucleated cells as per a case series), extensive skeletal involvement, high-risk cytogenetics, and plasmablastic morphology $[35,46]$. There are reports of bortezomib, thalidomide, and steroid associated TLS [23, 38]. Tumor lysis syndrome manifests due to lysis of massive numbers of tumor cells releasing intracellular potassium and phosphorous causing hyperkalemia and hyperphosphatemia. This causes calcium to precipitate as calcium phosphate in tissues causing secondary hypocalcemia. Hyperuricemia results from the breakdown of nucleic acids in the tumor cells. The resultant metabolic derangement, if left untreated, can progress to kidney failure, mental status changes, seizures, arrhythmias, and even death. Diagnosing TLS can be challenging in patients with MM due to its uncommon association and often seen associated renal insufficiency [131]. It is imperative for clinicians to be aware of this complication in MM patients undergoing treatment with novel combinations. TLS is managed with aggressive intravenous hydration, rasburicase, allopurinol, and at times sodium bicarbonate. Timely initiation of renal replacement therapy is paramount in patients with lifethreatening electrolyte disturbances.

\section{Nervous System}

Epidural spinal cord compression (ESCC): Acute emergencies related to the nervous system in $\mathrm{MM}$ are mostly related to ESCC. Vertebral involvement is prevalent in $60-80 \%$ of $\mathrm{MM}$ patients, and ESCC is encountered in approximately $10-20 \%$ of patients either at presentation or during the course of disease $[64,130]$. The compression usually results from vertebral fractures caused by osteolytic tumors or malignant osteoporosis. Very rarely, it has been reported due to extraosseous epidural MM or from extradural extension of plasmacytoma [124].

In majority of the cases, thoracic or lumbar spinal cord is involved $(>80 \%)[31,66]$. It is vital to recognize the symptoms early, with prompt imaging since the strongest predictor of neurologic outcome with treatment is the neurologic status when treatment is initiated [77]. Clinical features depend on the site and degree of involvement. A new-onset back pain or a worsening of an existing pain in a MM patient should prompt a work-up to rule this out. Pain can be localized or radiating which usually worsens with lying down. 
Associated bowel and bladder dysfunction is present in about half of the cases [44]. If treatment is delayed, myelopathy progresses to loss of sensory and motor function leading to paraplegia. Spinal cord injury at the cervical level is uncommon; however, if affected above $\mathrm{C} 4-\mathrm{C} 5$, it results in respiratory, cardiac, and autonomic decompensation [86]. Gadolinium-enhanced MRI is the initial gold standard imaging modality as it can aid in the thorough evaluation of spine, degree of tumor or fracture involvement, and for radiation planning [59]. The safety of MRI should be discussed in the presence of pacemakers and defibrillators in a multidisciplinary fashion, and if contraindicated, CT myelography should be performed.

There is a lack of consensus guidelines for treatment and hence warrants an emergent multidisciplinary team consult involving the oncologist, neurosurgeon, and radiation oncologist. The optimal management depends on the extent of disease, performance status of the patient, and presence of spine instability. Immediate therapeutic strategies involve achieving minimal mobility until stability of the spine is achieved. Steroids should be initiated even while awaiting diagnostic study results (dexamethasone at doses $10 \mathrm{mg}$ IV followed by $16 \mathrm{mg}$ daily in divided doses with a taper over 2 weeks) [137]. Doses as high as $100 \mathrm{mg}$ initial bolus were used in the past, but are not used any more due to risk of significant side effects and limited evidence to suggest benefit [45]. Spine stabilization by reducing the mechanical load can be achieved with the use of a neck collar (e.g., Philadelphia collar) in case of cervical spine or braces at other sites and is not generally recommended for longer than 3 months [87]. A thorough baseline neurological exam followed by serial neurological exams should be done to assess clinical status.

Radiotherapy should be started as soon as possible as MM is extremely radiosensitive, unless in very late in the disease. In a retrospective study which included 63 patients with MM/lymphoma treated with RT alone, $76 \%$ of the patients experienced an improvement in motor function, $22 \%$ had no change, while $2 \%$ deteriorated. In this group, patients who experienced slowly progressive motor deficit defined as time of onset
$>14$ days had better response rates than patients who had fast onset deficit defined as time of onset $<14$ days. The improvements in motor function in these groups were $80 \%$ and $70 \%$, respectively. No patients who received radiotherapy progressed in the slow onset motor weakness group [103, 104]. Current evidence does not support resorting to surgery in patients with ESCC, as most MM cases respond to radiation. The need for surgical stabilization with fixation or by percutaneous repair is in general reserved for cases with spine instability or spine compression from a vertebral body. Treatment of the underlying malignancy with systemic chemotherapy or novel agents, bone-modifying agents, and neurorehabilitation can help in recovery of the vertebral and spinal damage [72, 101].

CNS myelomatosis: Neurological symptoms in MM are most commonly due to hypercalcemia, cord compression, anti-MM drug related, hyperviscosity, or amyloid-related neuropathy. Direct involvement by malignant cells of the central nervous system (CNS) or peripheral nervous system, unlike lymphoma, is uncommon in MM. Direct CNS involvement in $\mathrm{MM}$ often presents as leptomeningeal myelomatosis (LMM), intraparenchymal metastasis, cranial nerve involvement, or radiculopathy $[54,95,117]$. Though conventional wisdom would suggest CNS involvement is seen later during the disease, most patients present relatively early with median time of diagnosis of LMD around 9 months from MM diagnosis. Hence the risk is unlikely only related to improved survival in MM patients but also due to an aggressive early biology. Supporting this contention, CNS involvement is reported mostly in patients with high-risk MM with heavy tumor burden, plasmablastic morphology, or plasma cell leukemia [138]. Patients often present with focal weakness, cranial nerve palsies, mental status changes, speech and gait disturbances, symptoms of increased intracranial pressure, and/or seizures. CSF examination reveals pleocytosis, increased protein levels higher than $100 \mathrm{~g} / \mathrm{dL}$, presence of monoclonal plasma cells, and in some cases an increased opening pressure. The diagnosis is usually reached with the first analysis of CSF 
[78]. MRI imaging with and without contrast would aid in identifying the presence and extent of the disease. In the presence of symptoms, steroids can help in alleviating the symptoms and reducing intracranial pressure. Therapy involves strategies similar to that used for other hematological malignancies with CNS involvement, including intrathecal chemotherapy and cranial irradiation with systemic chemotherapy. Despite majority of patients achieving CSF clearance with CNS-directed therapy showing significant potential for symptom improvement, the overall prognosis is grim with the currently approved therapies in MM. The median survival ranges from 3 to 6 months, with a small fraction surviving past 2 years [75]. Hence it is paramount to have a thorough discussion on the goals of therapy once CNS involvement is diagnosed [17, 34].

\section{Hematologic}

Anemia: Hematological emergencies in MM most commonly arise due to disease- or treatment-related myelosuppression. Anemia $(\mathrm{Hb}>2 \mathrm{~g} / \mathrm{dL}$ below the lower limit of normal or a hemoglobin value $<10 \mathrm{~g} / \mathrm{dL}$ ) is a common complication seen in $70-80 \% \mathrm{MM}$ patients at presentation and in nearly all patients at some point in the disease course [64]. Several factors drive anemia in MM patients which include bone marrow involvement, low serum erythropoietin level due to kidney dysfunction, hemolysis, impaired availability of storage iron, and anti-MM treatmentrelated bone marrow suppression. An aggressive anti-MM treatment approach despite the severity of anemia is warranted in patients with anemia suspected to be from MM. This is likely encountered in most cases of newly diagnosed MM. Myeloma therapy often worsens the anemia, before it improves once myeloma responds. These patients should be closely monitored for transfusion requirement. No universal guidelines exist for transfusion goals. In general, leukoreduced and irradiated blood is used for transfusion for hemoglobin level $<7-8 \mathrm{~g} / \mathrm{dL}$ or symptomatic anemia. The transfusion goal can vary in the presence of other comorbidities such as cardiac ischemia, active bleeding, or if symptoms due to hypoperfusion are present. A major proportion of patients with MM who have chronic anemia are those with long-standing disease in partial remission or stable response. Erythropoiesisstimulating agents are not routinely used, except in this population after careful selection, to improve the ongoing anemia despite chemotherapy.

Anti-MM drug-induced TMA: In patients who present with new-onset anemia and thrombocytopenia, more than often, it is ascribed to myelosuppressive treatment or for MM progression. In the setting of worsening AKI, it is imperative to check for evidence of hemolysis with a haptoglobin level, LDH level, and peripheral blood smear looking for schistocytes. One should strongly consider ruling out TMA as it has been reported with novel drugs and with older chemotherapeutic agents; two notable novel drugs are bortezomib and carfilzomib, and a chemotherapeutic agent is cisplatin. Prompt discontinuation of these medications is warranted if the medication is suspected to be the likely culprit. Plasmapheresis is initiated in most of patients, and eculizumab has been tried in a small number of patients. The role of these two therapies in malignancy-associated TMA is controversial and should be discussed on a case-by-case basis [51, $102,121,140]$.

\section{Conclusion}

The novel drug combinations with manageable adverse profile hold great promise in MM, which has turned MM into a chronic disease with an acceptable quality of life for patients. It is also crucial to recognize the complications of MM or its treatment and understand the management strategies, which could help prevent excess morbidity or mortality associated with this incurable disease.

Conflicts of Interest KP $=$ research funds from Poseida, Intrexon/Ziopharm, Takeda. Advisory board/consultancy in Takeda, DAVA oncology, Celgene, Janssen, Amgen, BMS, and Oncopeptides. 


\section{References}

1. Abbott KC, Agodoa LY. Multiple myeloma and light chain-associated nephropathy at end-stage renal disease in the United States: patient characteristics and survival. Clin Nephrol. 2001;56(3):207-10.

2. Afessa B, Tefferi A, et al. Diffuse alveolar hemorrhage in hematopoietic stem cell transplant recipients. Am J Respir Crit Care Med. 2002;166(5):641-5.

3. Ansari-Lari MA, Ali SZ. Fine-needle aspiration of abdominal fat pad for amyloid detection: a clinically useful test? Diagn Cytopathol. 2004;30:178-81.

4. Ash-Bernal R, Browner I, Erlich R. Early detection and successful treatment of drug-induced pneumonitis with corticosteroids. Cancer Invest. 2002; 20(7-8):876-9.

5. Atrash S, Tullos A, et al. Cardiac complications in relapsed and refractory multiple myeloma patients treated with carfilzomib. Blood Cancer J. 2015;5(1): e272.

6. Augustson BM, Begum G, et al. Early mortality after diagnosis of multiple myeloma: analysis of patients entered onto the United Kingdom Medical Research Council trials between 1980 and 2002 - Medical Research Council Adult Leukaemia Working Party. J Clin Oncol. 2002;23:9219-26.

7. Ayed AO, Moreb JS, et al. Severe diffuse alveolar hemorrhage associated with bortezomib administration in patients with multiple myeloma. Am Soc Hematol. 2014;5761-5761.

8. Wirk B. Bortezomib-related diffuse alveolar hemorrhage. J Clin Oncol. 2012;30(36):e379-81.

9. Bellomo R, Ronco C, et al. Acute renal failure-definition, outcome measures, animal models, fluid therapy and information technology needs: the Second International Consensus Conference of the Acute Dialysis Quality Initiative (ADQI) Group. Crit Care. 2004;8(4):R204.

10. Bhogal S, Ladia V, et al. Cardiac amyloidosis: an updated review with emphasis on diagnosis and future directions. Curr Probl Cardiol. 2018;43(1): 10-34.

11. Bladé J, Fernández-Llama P, et al. Renal failure in multiple myeloma: presenting features and predictors of outcome in 94 patients from a single institution. Arch Intern Med. 1998;158(17):1889-93.

12. Bladé J, Rosiñol L. Renal, hematologic and infectious complications in multiple myeloma. Best Pract Res Clin Haematol. 2005;18(4):635-52.

13. Blom JW, Doggen CJ, et al. Malignancies, prothrombotic mutations, and the risk of venous thrombosis. JAMA. 2005;293(6):715-22.

14. Bridoux F, Pegourie B, Augeul-Meunier K, et al. Treatment of myeloma cast nephropathy $(\mathrm{MCN})$ : a randomized trial comparing intensive haemodialysis (HD) with high cut-off (HCO) or standard high-flux dialyzer in patients receiving a bortezomib-based regimen (the MYRE Study, by the Intergroupe
Francophone du Myélome (IFM) and the French Society of Nephrology (SFNDT)). Blood. 2016;128: 978.

15. Bridoux F, Sirac C, et al. Fanconi's syndrome induced by a monoclonal Vк3 light chain in Waldenström's macroglobulinemia. Am J Kidney Dis. 2005;4(45): 74.

16. Buttin BM, Moore MJ. Thalidomide-induced reversible interstitial pneumonitis in a patient with recurrent ovarian cancer. Gynecol Oncol. 2008;111(3):546-8.

17. Cerase A, Tarantino A, et al. Intracranial involvement in plasmacytomas and multiple myeloma: a pictorial essay. Neuroradiology. 2008;50(8):665-74.

18. Chang H, Lee SY, Tang TC. Tumor lysis syndrome in patients with light chain multiple myeloma: report of two cases. Chang Gung Med J. 2011;34(6 Suppl): 70-5.

19. Chang JT, Green L, Beitz J. Renal failure with the use of zoledronic acid. N Engl J Med. 2003;349:1676-9.

20. Chen Y, Kiatsimkul P, et al. Lenalidomide-induced interstitial lung disease. Pharmacotherapy. 2010; 30(3):325.

21. Clark WF, Stewart AK, et al. Plasma exchange when myeloma presents as acute renal failure: a randomized, controlled trial. Ann Intern Med. 2005; 143(11):777-84.

22. Cook M, Hutchison C, Fifer L, Gillmore J, Heyne N, Weisel K, et al. High cut-off haemodialysis (HCO-HD) does not improve outcomes in myeloma cast nephropathy: results of European trial of Free Light Chain removal by extended haemodialysis in cast nephropathy. EHA. 2016; Abstract P270.

23. Chim CS. Rapid complete remission in multiple myeloma with bortezomib/thalidomide/dexamethasone combination therapy following development of tumor lysis syndrome. Cancer Chemother Pharmacol. 2008;62(1):181-2.

24. Scholz DA. Effect of steroid therapy on hypercalcemia and renal insufficiency in sarcoidosis. J Am Med Assoc. 1959;169(7):682-7.

25. De Lassence A, Fleury-Feith J, et al. Alveolar hemorrhage. Diagnostic criteria and results in 194 immunocompromised hosts. Am J Respir Crit Care Med. 1995;151(1):157-63.

26. den Exter PL, Kooiman J, et al. New anticoagulants in the treatment of patients with cancer-associated venous thromboembolism. Best Pract Res Clin Haematol. 2013;26(2):163-9.

27. Di Pumpo M, Ponziano E, et al. Metabolic disorders in hematologic malignancies-A review. iMedPub J. 2015;7(5):14.

28. Dimopoulos M, Kyle R, et al. Guidelines for standard investigative workup: report of the International Myeloma Workshop Consensus Panel 3. Blood. 2011;117(18):4701-4705.

29. Dimopoulos MA, Terpos E, et al. Renal impairment in patients with multiple myeloma: a consensus statement on behalf of the International Myeloma Working Group. J Clin Oncol. 2010;28(33):4976-84. 
30. Dimopoulos MA, Richardson PG, et al. VMP (Bortezomib, Melphalan, and Prednisone) is active and well tolerated in newly diagnosed patients with multiple myeloma with moderately impaired renal function, and results in reversal of renal impairment: cohort analysis of the phase III VISTA study. J Clin Oncol. 2009;27:6086-93.

31. Drappatz J, Batchelor T. Neurologic complications of plasma cell disorders. Clin Lymphoma. 2004; 5(3):163-71.

32. Dubrey SW, Cha K, et al. The clinical features of immunoglobulin light-chain (AL) amyloidosis with heart involvement. QJM. 1998;91:141-57.

33. Elice F, Fink L, et al. Acquired resistance to activated protein $\mathrm{C}$ (aAPCR) in multiple myeloma is a transitory abnormality associated with an increased risk of venous thromboembolism. Br J Haematol. 2006; 134:399.

34. Fassas AB, Ward S, et al. Myeloma of the central nervous system: strong association with unfavorable chromosomal abnormalities and other high-risk disease features. Leuk Lymphoma. 2004;45(2):291.

35. Fassas AB, Desikan KR, et al. Tumour lysis syndrome complicating high-dose treatment in patients with multiple myeloma. Br J Haematol. 1999; 105(4):938-41.

36. Feng D, Syed IS, et al. Intracardiac thrombosis and anticoagulation therapy in cardiac amyloidosis. Circulation. 2009;119(18):2490-7.

37. Fizazi K, Carducci M, et al. Denosumab versus zoledronic acid for treatment of bone metastases in men with castration-resistant prostate cancer: a randomised, double-blind study. Lancet. 2011; 377(9768):813-22.

38. Fuente N, Mañe JM, et al. Tumor lysis syndrome in a multiple myeloma treated with thalidomide. Ann Oncol. 2004;15(3):537.

39. Clines GA. Mechanisms and treatment of hypercalcemia of malignancy. Curr Opin Endocrinol Diabetes Obes. 2011;18(6):339-46.

40. Garewal H, Durie B. Aggressive phase of multiple myeloma with pulmonary plasma cell infiltrates. JAMA. 1982;248(15):1875-6.

41. Gertz MA, Skinner M, et al. Selective binding of nifedipine to amyloid fibrils. Am J Cardiol. 1985; 55(13): 1646.

42. Golde DW, Drew WL, et al. Occult pulmonary haemorrhage in leukaemia. BMJ. 1975;2:166-8.

43. Gonsalves WI, Leung N, et al. Improvement in renal function and its impact on survival in patients with newly diagnosed multiple myeloma. Blood Cancer J. 2015;5:e296.

44. Gonzalez-Osete G, Modiano M. Spinal cord compression. In: Decision making in medicine. 1993. p. 292. Mosby Elsevier, Philadelphia.

45. Graham PH, Capp A, et al. A pilot randomised comparison of dexamethasone $96 \mathrm{mg}$ vs $16 \mathrm{mg}$ per day for malignant spinal-cord compression treated by radiotherapy: TROG 01.05 Superdex study. Clin Oncol. 2006;18(1):70-6.

46. Greipp PR, Leong T, et al. Plasmablastic morphology - an independent prognostic factor with clinical and laboratory correlates: Eastern Cooperative Oncology Group (ECOG) myeloma trial E9486 report by the ECOG Myeloma Laboratory Group. Blood. 1998;91: 2501-7.

47. Grogan M, Dispenzieri A, Gertz MA. Light-chain cardiac amyloidosis: strategies to promote early diagnosis and cardiac response. Heart. 2017. https://doi. org/10.1136/heartjnl-2016-310704.

48. Hamon D, Algalarrondo V, et al. Outcome and incidence of appropriate implantable cardioverterdefibrillator therapy in patients with cardiac amyloidosis. Int J Cardiol. 2016;222:562-8.

49. Kwaan HC. Hyperviscosity in plasma cell dyscrasias. Clin Hemorheol Microcirc. 2013;55:75-83.

50. Henry DH, Costa L, et al. Randomized, double-blind study of denosumab versus zoledronic acid in the treatment of bone metastases in patients with advanced cancer (excluding breast and prostate cancer) or multiple myeloma. J Clin Oncol. 2011;29: 1125-32.

51. Hobeika L, Self SE, Velez JC. Renal thrombotic microangiopathy and podocytopathy associated with the use of carfilzomib in a patient with multiple myeloma. BMC Nephrol. 2014;15(1):156.

52. Honton B, Despas F, et al. Bortezomib and heart failure: case-report and review of the French Pharmacovigilance database. Fundam Clin Pharmacol. 2014;28:349-52.

53. Hsu P, Lin TW, et al. Risk of early mortality in patients with newly diagnosed multiple myeloma. Medicine. 2015;94(50):e2305.

54. Husain MM, Metzer WS, Binet EF. Multiple intraparenchymal brain plasmacytomas with spontaneous intratumoral hemorrhage. Neurosurgery. 1987; 20(4):619-23.

55. Hutchison CA, Bradwell AR, et al. Treatment of acute renal failure secondary to multiple myeloma with chemotherapy and extended high cut-off hemodialysis. Clin J Am Soc Nephrol. 2009;4(4):745-54.

56. Hutchison CA, Batuman V, et al. The pathogenesis and diagnosis of acute kidney injury in multiple myeloma. Nat Rev Nephrol. 2012;8(1):43.

57. Hutchison CA, Cockwell P, et al. Early reduction of serum-free light chains associates with renal recovery in myeloma kidney. J Am Soc Nephrol. 2011. https:// doi.org/10.1681/ASN.2010080857.

58. Jacobson DR, Zolla-Pazner S. Immunosuppression and infection in multiple myeloma. Semin Oncol. 1986;13:282-90.

59. Jung HS, Jee WH, et al. Discrimination of metastatic from acute osteoporotic compression spinal fractures with MR imaging. Radiographics. 2003;23(1): 179-87. 
60. Kamble R, Rosenzweig T. Diffuse pulmonary parenchymal involvement in multiple myeloma: antemortem diagnosis. Int J Hematol. 2006;83(3):259.

61. Kang W, Kim JS, et al. Nonspecific interstitial pneumonitis after bortezomib and thalidomide treatment in a multiple myeloma patient. Yonsei Med J. 2010; 51(3):448-50.

62. Kim YJ, Kim SJ, et al. Multiple myeloma with myelomatous pleural effusion: a case report and review of the literature. Acta Haematol. 2008;120(2):108-11.

63. Kumar A, Djulbegovic B, Soares HP. Plasmapheresis in the treatment of renal failure associated with multiple myeloma. Am Soc Hematology. 2006;3585.

64. Kyle RA, Gertz MA, et al. Review of 1027 patients with newly diagnosed multiple myeloma. Mayo Clin Proc. 2003;78:21-33.

65. Wisneski LA. Salmon calcitonin in the acute management of hypercalcemia. Calcif Tissue Int. 1990;46:26-30.

66. Lecouvet FE, Vande Berg BC, et al. Vertebral compression fractures in multiple myeloma. Part I. Distribution and appearance at MR imaging. Radiology. 1997;204(1):195-9.

67. Lee AY, Levine MN, et al. Low-molecular-weight heparin versus a coumarin for the prevention of recurrent venous thromboembolism in patients with cancer. N Engl J Med. 2003;349:146-53.

68. LeGrand SB, Leskuski D, Zama I. Narrative review: furosemide for hypercalcemia: an unproven yet common practice. Ann Intern Med. 2008;149(4):259-63.

69. Libbey CA, Skinner M, Cohen AS. Use of abdominal fat tissue aspirate in the diagnosis of systemic amyloidosis. Arch Intern Med. 1983;143:1549-52.

70. Liberman V, D'Agati VD, et al. Acute tubular necrosis in a patient with myeloma treated with carfilzomib. Kidney Int Rep. 2016;1(2):89-92.

71. Lin G, Dispenzieri A, et al. Implantable cardioverter defibrillators in patients with cardiac amyloidosis. J Cardiovasc Electrophysiol. 2013;24(7):793-8.

72. Loblaw DA, Mitera G, et al. A 2011 updated systematic review and clinical practice guideline for the management of malignant extradural spinal cord compression. Int J Radiat Oncol Biol Phys. 2012; 84(2):312-7.

73. Lok R, Golovyan D, Smith J. Multiple myeloma causing interstitial pulmonary infiltrates and softtissue plasmacytoma. Respir Med Case Rep. 2018;24:155-7.

74. Ludwig H, Durie BG, et al. Myeloma in patients younger than age 50 years presents with more favorable features and shows better survival: an analysis of 10549 patients from the International Myeloma Working Group. Blood. 2008;111(8):4039-47.

75. Majd N, Demopoulos A, Chari A. Central nervous system involvement in multiple myeloma patients in the era of novel therapies (P07.005). Neurology. 2013;80(7 Suppl):P07-005.

76. Major P, Lortholary A, et al. Zoledronic acid is superior to pamidronate in the treatment of hypercalcemia of malignancy: a pooled analysis of two randomized, controlled clinical trials. J Clin Oncol. 2001; 19(2):558-67.

77. Maranzano E, Latini P. Effectiveness of radiation therapy without surgery in metastatic spinal cord compression: final results from a prospective trial. Int J Radiat Oncol Biol Phys. 1995;32(4):959-67.

78. Marjanović S, Mijusković Z, et al. Multiple myeloma invasion of the central nervous system. Vojnosanit Pregl. 2012;69(2):209-13.

79. Marković O, Marisavljević D, et al. Proliferative activity of myeloma cells determined by Ki-67 antibody: biological and clinical significance. Vojnosanit Pregl. 2005;62(1):33-8.

80. Marmor DB, Farber JL, Gottlieb JE. Acute respiratory distress syndrome due to pulmonary involvement by neoplastic plasma cells in multiple myeloma. Thorax. 2006;61(5):455-6.

81. Mehta J, Singhal S. Hyperviscosity syndrome in plasma cell dyscrasias. Semin Thromb Hemost. 2003;29:467-72.

82. Meltzer LE, Palmon FP Jr, et al. Acute pancreatitis secondary to hypercalcemia of multiple myeloma. Ann Intern Med. 1962;57(6):1008-12.

83. Menke MN, Feke GT, et al. Effect of plasmapheresis on hyperviscosity-related retinopathy and retinal hemodynamics in patients with Waldenstrom's macroglobulinemia. Invest Ophthalmol Vis Sci. 2008;49: 1157-60.

84. Mesquita ET, Jorge AJL, et al. Cardiac amyloidosis and its new clinical phenotype: heart failure with preserved ejection fraction. Arq Bras Cardiol. 2017;109:71-80.

85. Metcalf JP, Rennard SI, et al. Corticosteroids as adjunctive therapy for diffuse alveolar hemorrhage associated with bone marrow transplantation. Am J Med. 1994;96(4):327-34.

86. Meyer NJ, Davis SQ, Kress JP. Acute tachypnea during mechanical ventilation in a 62-year-old man with multiple myeloma involving the spinal cord. Chest. 2006;130(2):616.

87. Molloy S, Lai M, et al. Optimizing the management of patients with spinal myeloma disease. Br J Haematol. 2015;171(3):332-43.

88. Murakami H, Hayashi K, et al. Risk factors for early death in patients undergoing treatment for multiple myeloma. Ann Hematol. 2001;80(8):452.

89. Niesvizky R, Martínez-Baños D, et al. Prophylactic low-dose aspirin is effective antithrombotic therapy for combination treatments of thalidomide or lenalidomide in myeloma. Leuk Lymphoma. 2007;48(12):2330-7.

90. Nordlinger M, Magnani B, et al. Is elevated plasma B-natriuretic peptide in amyloidosis simply a function of the presence of heart failure? Am J Cardiol. 2005;96(7):982-4.

91. Nucci M, Anaissie E. Infections in patients with multiple myeloma in the era of high-dose therapy and novel agents. Clin Infect Dis. 2009;49:1211-25. 
92. Enrico O, Gabriele B, et al. Unexpected cardiotoxicity in haematological bortezomib treated patients. $\mathrm{Br}$ J Haematol. 2007;138:396-7.

93. Papandreou CN, Daliani DD, et al. Phase I trial of the proteasome inhibitor bortezomib in patients with advanced solid tumors with observations in androgenindependent prostate cancer. J Clin Oncol. 2004; 22(11):2108-21.

94. Park MS, Kim BC, et al. Cerebral infarction in IgG multiple myeloma with hyperviscosity. J Korean Med Sci. 2005;20(4):699-701.

95. Patriarca F, Zaja F, et al. Meningeal and cerebral involvement in multiple myeloma patients. Ann Hematol. 2001;80(12):758-62.

96. Pecherstorfer M, Herrmann Z, et al. Randomized phase II trial comparing different doses of the bisphosphonate ibandronate in the treatment of hypercalcemia of malignancy. J Clin Oncol. 1996;14(1): 268-76.

97. Pecherstorfer M, Steinhauer EU, et al. Efficacy and safety of ibandronate in the treatment of hypercalcemia of malignancy: a randomized multicentric comparison to pamidronate. Support Care Cancer. 2003;11(8):539.

98. Peigne V, Rusinová K, et al. Continued survival gains in recent years among critically ill myeloma patients. Intensive Care Med. 2009;35(3):512.

99. Poe RH, Kamath C, et al. Acute respiratory distress syndrome with pulmonary calcification in two patients with $\mathrm{B}$ cell malignancies. Respiration. 1989;56(1-2):127-33.

100. Pratt G, Goodyear O, Moss P. Immunodeficiency and immunotherapy in multiple myeloma. Br J Haematol. 2007;138(5):563-79.

101. Prestwich RJ, Ackroyd S, Gilson D. Is surgery required in the management of spinal cord compression in myeloma patients? Clin Oncol. 2011;23(2): 161.

102. Qaqish I, Schlam IM, et al. Carfilzomib: a cause of drug induced thrombotic microangiopathy. Transfus Apher Sci. 2016;54:401-4.

103. Rades D, Heidenreich F, Karstens JH. Final results of a prospective study of the prognostic value of the time to develop motor deficits before irradiation in metastatic spinal cord compression. Int J Radiat Oncol Biol Phys. 2002;53:975-9.

104. Rades D, Veninga T, et al. Outcome after radiotherapy alone for metastatic spinal cord compression in patients with oligometastases. J Clin Oncol. 2006; 25(1):50-6.

105. Rathi NK, Tanner AR, et al. Low-, medium- and highdose steroids with or without aminocaproic acid in adult hematopoietic SCT patients with diffuse alveolar hemorrhage. Bone Marrow Transplant. 2015; 50(3):420.

106. Ravinet A, Perbet S, et al. Lung postmortem autopsy revealing extramedullary involvement in multiple myeloma causing acute respiratory distress syndrome. Case Rep Hematol. 2014;2014:635237.
107. Rubinow A, Skinner M, Cohen AS. Digoxin sensitivity in amyloid cardiomyopathy. Circulation. 1981; 63(6):1285-8.

108. Rysava R, Lachmanová J, et al. O039 Dialysis with HCO membrane in combination with potent chemotherapy increases the probability for renal recovery and better survival in patients with acute renal failure due to myeloma kidney-data from Czech Republic. Nephrol Dial Transpl. 2016;31(1):i18. https://doi.org/ 10.1093/ndt/gfw124.01

109. Savage DG, Lindenbaum J, Garrett TJ. Biphasic pattern of bacterial infection in multiple myeloma. Ann Intern Med. 1982;96(1):47-50.

110. Schwartz J, Padmanabhan A, et al. Guidelines on the use of therapeutic apheresis in clinical practiceevidence-based approach from the Writing Committee of the American Society for Apheresis: the seventh special issue. J Clin Apher. 2016;31(3):149-338.

111. Sekine M, Takami H. Combination of calcitonin and pamidronate for emergency treatment of malignant hypercalcemia. Oncol Rep. 1998;5:197-9.

112. Selvanayagam JB, Hawkins PN, et al. Evaluation and management of the cardiac amyloidosis. J Am Coll Cardiol. 2007;50(22):2101-10.

113. Shahzad H, Goralnik J, et al. Lenalidomide induced interstitial lung disease. Drug induced lung disease: case reports. Am Thorac Soc. 2017; A5551.

114. Shaikh BS, Lombard RM, et al. Changing patterns of infections in patients with multiple myeloma. Oncology. 1982;39:78-82.

115. Shimanovsky A, Alvarez Argote J, et al. Autoimmune manifestations in patients with multiple myeloma and monoclonal gammopathy of undetermined significance. BBA Clin. 2016;6:12-8.

116. Siegel D, Bilotti E, van Hoeven KH. Serum free light chain analysis for diagnosis, monitoring, and prognosis of monoclonal gammopathies. Lab Med. 2009; 40(6):363-6.

117. Slager UT, Taylor WF, et al. Leptomeningeal myeloma. Arch Pathol Lab Med. 1979;103(13):680-2.

118. Soubani AO, Miller KB, Hassoun PM. Pulmonary complications of bone marrow transplantation. Chest. 1996;109(4):1066-77.

119. Stone MJ, Bogen SA. Evidence-based focused review of management of hyperviscosity syndrome. Blood. 2012;119(10):2205-8.

120. Sugita Y, Ohwada C, et al. Early-onset severe diffuse alveolar hemorrhage after bortezomib administration suggestive of pulmonary involvement of myeloma cells. J Clin Exp Hematop. 2015;55(3):163-8.

121. Sullivan MR, Danilov AV, et al. Carfilzomib associated thrombotic microangiopathy initially treated with therapeutic plasma exchange. J Clin Apher. 2015;30:308-10.

122. Teh BW, Teng JC, et al. Invasive fungal infections in patients with multiple myeloma: a multi-center study in the era of novel myeloma therapies. Haematologica. 2015;100(1):e28-31. 
123. Thornburg A, Abonour R, et al. Hypersensitivity pneumonitis-like syndrome associated with the use of lenalidomide. Chest. 2007;131(5):1572-4.

124. Byrne TN. Spinal cord compression from epidural metastases. N Engl J Med. 1992;327(9):614-9.

125. Tsakiris DJ, Stel VS, et al. Incidence and outcome of patients starting renal replacement therapy for end-stage renal disease due to multiple myeloma or light-chain deposit disease: an ERA-EDTA Registry study. Nephrol Dial Transplant. 2009;25(4):1200-6.

126. vanden Driesen RI, Slaughter RE, Strugnell WE. MR findings in cardiac amyloidosis. Am J Roentgenol. 2006;186(6):1682-5.

127. Varr BC, Zarafshar S, et al. Implantable cardioverterdefibrillator placement in patients with cardiac amyloidosis. Heart Rhythm. 2014;11(1):158-62.

128. Voortman J, Giaccone G. Severe reversible cardiac failure after bortezomib treatment combined with chemotherapy in a non-small cell lung cancer patient: a case report. BMC Cancer. 2006;6(1):129.

129. Wada S, Yasuda S, et al. Regulation of calcitonin receptor by glucocorticoid in human osteoclast-like cells prepared in vitro using receptor activator of nuclear factor- $\kappa \mathrm{B}$ ligand and macrophage colonystimulating factor. Endocrinology. 2001;142(4): 1471-8.

130. Wallington M, Mendis S, et al. Local control and survival in spinal cord compression from lymphoma and myeloma. Radiother Oncol. 1997;42(1):43-7.

131. Wang L, Jian Y, et al. Management of tumor lysis syndrome in patients with multiple myeloma during bortezomib treatment. Clin J Oncol Nurs. 2015;19:E4.

132. Wang M, Weber DM, et al. Thalidomidedexamethasone as primary therapy for advanced multiple myeloma. Am J Hematol. 2005;79(3):194-7.
133. Waxman AJ, Clasen S, et al. Carfilzomib-associated cardiovascular adverse events: a systematic review and meta-analysis. JAMA Oncol. 2018;4(3): e174519.

134. Wei Ma AT, Cheuk Lam Lo R, et al. Multiple myeloma presenting as interstitial lung disease. J Clin Oncol. 2010;29(6):e143-4.

135. Willis MS, Patterson C. Into the heart: the emerging role of the ubiquitin-proteasome system. J Mol Cell Cardiol. 2006;41:567-79.

136. Xiao Y, Yin J, et al. Incidence and risk of cardiotoxicity associated with bortezomib in the treatment of cancer: a systematic review and metaanalysis. PLoS One. 2014;9(1):e87671.

137. Yamada KA, Awadalla S. Acute spinal cord dysfunction. In: The Washington manual of medical therapeutics. 31st ed. Philadelphia: Lippincott Williams \& Wilkins; 2004.

138. Yellu MR, Engel J, et al. Retracted: overview of recent trends in diagnosis and management of leptomeningeal multiple myeloma. Hematol Oncol. 2016;34(1):2-3.

139. Yuan Y, Wieczorek R, et al. Multiple myeloma involving skin and pulmonary parenchyma after autologous stem cell transplantation. J Hematol Oncol. 2009;2(1):48.

140. Yui JC, Van Keer J, et al. Proteasome inhibitor associated thrombotic microangiopathy. Am J Hematol. 2016;91:E348-52.

141. Zagouri F, Kastritis E, et al. Hypercalcemia remains an adverse prognostic factor for newly diagnosed multiple myeloma patients in the era of novel antimyeloma therapies. Eur J Haematol. 2017;99(5): 409-14. 